\title{
Optimal Path Planning for Unmanned Combat Aerial Vehicles to Defeat Radar Tracking
}

\author{
Pierre T. Kabamba, ${ }^{*}$ Semyon M. Meerkov, ${ }^{\dagger}$ and Frederick H. Zeitz III ${ }^{\ddagger}$ \\ University of Michigan, Ann Arbor, Michigan 48109
}

\begin{abstract}
The problem of path planning for unmanned combat aerial vehicles (UCAVs) in the presence of radar-guided surface-to-air missiles is treated. The problem is formulated in the framework of the interaction between three subsystems: the aircraft, the radar, and the missile. The main features of this integrated model are as follows. The aircraft radar cross section (RCS) depends explicitly on both the aspect and bank angles; hence, the RCS and aircraft dynamics are coupled. The probabilistic nature of radar tracking is accounted for, namely, the probability that the aircraft has been continuously tracked depends on the aircraft RCS and range. Finally, the requirement to maintain tracking before missile launch and during missile flyout are also modeled. Based on this model, the problem of UCAV path planning is formulated as a minimax optimal control problem, with the aircraft lateral acceleration serving as control. Necessary conditions of optimality for this minimax problem are derived and used as a basis for an efficient numerical solution. Illustrative examples are considered that confirm the standard flying tactics of "denying range, aspect, and aim," by yielding flight paths that weave to avoid long exposures of aspects with large RCS.
\end{abstract}

\section{Introduction}

$\mathbf{T}$ HIS paper is devoted to the problem of automated path planning for unmanned combat aerial vehicles (UCAVs) in the presence of radar-guided surface-to-air missiles (SAMs). This problem features the interaction between three subsystems: the aircraft and its characteristics, the radar and its capabilities, and the missile and its lethality. Therefore, the solution of the UCAV path-planning problem requires realistic models of these three subsystems. Although the current literature offers models for each of them separately, there is no approach that integrates models of the three subsystems in a unified framework. The purpose of this paper is to propose such an integrated model and present results on its use.

The UCAV considered in this paper is distinguished from the unmanned aerial vehicle (UAV) by the mission it flies. Specifically, the UCAV mission, such as suppression of enemy air defense, may dictate more exposure to the SAM threat than a reconnaissance mission flown by a UAV. The current literature does not distinguish between mission types and uses the term UAV to refer to any unmanned aerial vehicle. However, this paper addresses the problem of planning for the high-threat mission and, therefore, uses the term UCAV.

Although the current literature on UAV path planning does not contain models that integrate aircraft, radar, and missile subsystems, a vast literature is available on each of these subsystems separately. A brief review of standard relevant results in this literature is as follows. Modeling of aircraft dynamics and radar cross section (RCS) have been widely discussed, for example, see Refs. 1-15 and the references therein. In particular, the dependence of aircraft RCS on aspect and bank angles is a well-documented phenomenon. ${ }^{16-18}$ Aircraft detection by a radar is treated as a random event depending on the signal-to-noise ratio of the radar return. ${ }^{19-21}$ Aircraft tracking by a radar after detection and missile guidance to interception have been discussed in Refs. 22-25. The focus of

Received 28 October 2004; revision received 5 February 2005; accepted for publication 7 February 2005. Copyright (C) 2005 by the American Institute of Aeronautics and Astronautics, Inc. All rights reserved. Copies of this paper may be made for personal or internal use, on condition that the copier pay the $\$ 10.00$ per-copy fee to the Copyright Clearance Center, Inc., 222 Rosewood Drive, Danvers, MA 01923; include the code 0731-5090/06 $\$ 10.00$ in correspondence with the CCC.

*Professor, Department of Aerospace Engineering, 1320 Beal Avenue.

${ }^{\dagger}$ Professor, Department of Electrical Engineering and Computer Science, 1301 Beal Avenue.

${ }^{\ddagger}$ Graduate Student, Department of Electrical Engineering and Computer Science, 1301 Beal Avenue. the literature in this area is on the analysis and design of radar systems.

The literature on UAV path planning can be divided in two groups. The first group treats the problem under the assumption of isotropic, that is, independent of aspect and bank angles, RCS, whereas the second assumes nonisotropic RCS. Representative publications in the first group include Refs. 1-11. Specifically, in Refs. 1-3, results are presented on minimizing the total reflected energy received by the radar, whereas in Refs. 4-7 using Voronoi diagrams for threat avoidance is suggested. In Refs. 8-10 the method of singular perturbations is used, and in Ref. 11 wavelets are used to address threedimensional path planning, but without referring to variations in RCS with aspect and bank angles. Coordinated planning for multiple aircraft is treated in Refs. 2, 6, 7, and 11 using optimal resource allocation techniques.

UAV path-planning problems with nonisotropic RCSs are considered in Refs. 12-15. Of these, in Ref. 12, a potential field is utilized in the presence of multiple radars but a bank-to-turn model is not used; in Ref. 13, both aircraft bank and yaw angles are optimized within aerodynamic restrictions for a given route; in Ref. 14, virtual forces are used but these forces are modified to account for varying RCS; and in Ref. 15, the detection probability is modeled as being dependent on azimuth, elevation, and slant range, but a bank-to-turn model is not used. Hence, with the exception of Refs. 13 and 14, none of the cited references account for the coupling between the RCS and dynamics through the aspect and bank angles. Moreover, only in Ref. 15 is the probabilistic nature of aircraft detection by a radar taken into account.

The recent work of Misovec et al. ${ }^{15}$ merits special mention. They treat path planning as a nonlinear optimization problem and present numerical solutions. They feature a nonlinear trajectory-generation method, based on sequential quadratic programming, which has the capability to model temporal constraints. This allows the authors to account for a condition called lock-loss, in which the radar system loses track of the target after a specified time of no detection. Although the model used in this work is quite accurate, it does not use bank-to-turn kinematics and, therefore, does not account for coupling between the RCS and aircraft dynamics.

The present work addresses this and other issues. Specifically, the integrated model of the aircraft, radar, and missile subsystems presented here has the following original features. First, the aircraft RCS depends on both the aspect and bank angles. Moreover, the turn rate of the aircraft is determined by its bank angle. Hence, the RCS and aircraft dynamics are coupled through the aspect and bank angles. Second, the probabilistic nature of radar tracking is considered. 
Specifically, a conservative estimate of the probability of tracking is derived from the aircraft RCS and range. Third, the decision process for launching a SAM and the requirement to maintain tracking during missile flyout are also included. Specifically, the probability that the UCAV is shot down is bounded from above by the probability of tracking, averaged over a time interval of length $T_{\text {resp }}+T_{\text {fo }}$. Here, $T_{\text {resp }}$ is the response time of the radar and $T_{\text {fo }}$ the flyout time of the missile.

Based on this integrated model, the problem of UCAV path planning is formulated as a minimax optimal control problem, with moving average functional, in which the aircraft lateral acceleration serves as control. The current paper treats this optimization problem and provides the following original contributions. Necessary conditions of optimality for the minimax optimal control problem with moving average functional are formulated. Based on these necessary conditions, properties of the optimal paths are derived. Finally, an efficient numerical optimization procedure is proposed that uses the described properties of optimal paths.

Our results on UCAV path planning using this integrated model are encouraging in that they confirm standard flying tactics. Indeed, they suggest that, in the presence of SAM threats, an aircraft should "deny range, aspect, and aim." 26

The remainder of the paper is as follows. In Sec. II, our model is presented. In Sec. III the dynamic optimization problem is formulated for threat avoidance. In Sec. IV, the necessary conditions of optimality are used to characterize the optimal control, whereas in Sec. V the qualitative properties of optimal paths using these optimal controls are discussed. In Sec. VI, an efficient numerical procedure that utilizes the properties of the optimal paths is described, whereas in Sec. VII our results with several examples are given. Section VII provides the conclusions. The necessary conditions of optimality for the minimax problem formulated in Sec. III are derived in Appendices A and B.

\section{Modeling}

In this section, the model we used throughout this paper is presented. The model consists of three subsystems: aircraft dynamics and RCS, radar tracking, and missile launch and lethality. We present in detail each of these subsystems.

\section{A. Aircraft Model}

The bank-to-turn aircraft is assumed to move in a horizontal plane at a constant altitude according to the equations

$$
\dot{x}=v \cos \psi, \quad \dot{y}=v \sin \psi, \quad \dot{\psi}=u / v, \quad|u|<U
$$

where $x$ and $y$ are the Cartesian coordinates of the aircraft, $\psi$ is the heading angle as shown in Fig. 1, $v$ is the constant speed, $u$ is the input signal and is the acceleration normal to the flight path vector, and $U$ is the maximum allowable lateral acceleration.

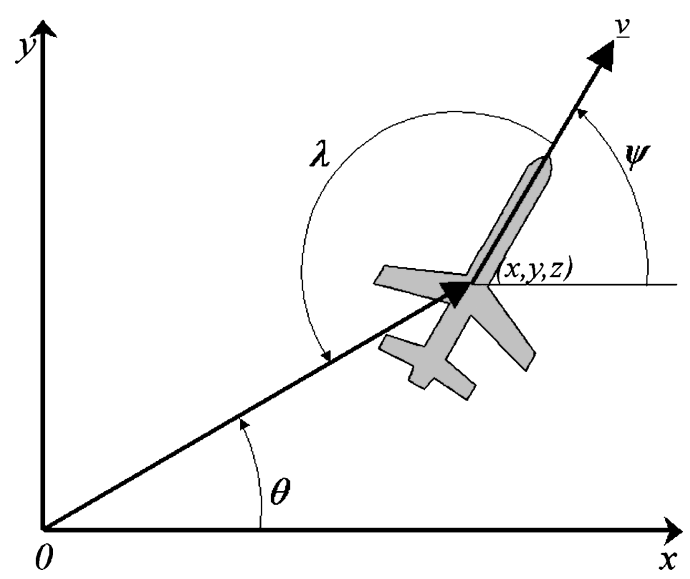

Fig. 1 Aircraft position, velocity, azimuth, heading, and aspect angles.

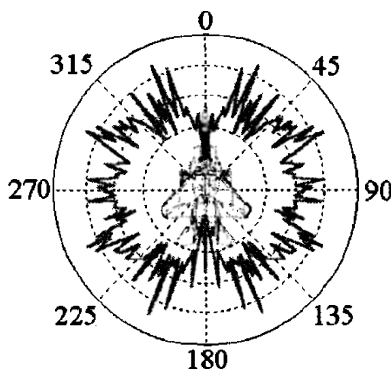

a) RCS as a function of aspect angle

Fig. 2 Dependence of RCS on aspect and bank angles.

Let

$$
\begin{gathered}
\theta=\arctan (y / x), \quad \lambda=\theta-\psi+\pi \\
\phi=\arctan \left(z / \sqrt{x^{2}+y^{2}}\right)
\end{gathered}
$$

be the azimuth, aspect, and elevation angles, respectively, where $z$ is the aircraft altitude. (See Fig. 1 for a projection onto the $x-y$ plane.) Let the bank angle $\mu$ be given by

$$
\mu=\arctan (u / g)
$$

where $g$ is the acceleration of gravity.

We model the RCS of the aircraft as a function of the aspect angle $\lambda$, the elevation angle $\phi$, and the bank angle $\mu$, so that

$$
\mathrm{RCS}=\sigma(\lambda, \phi, \mu)
$$

As an example, real aircraft RCS measurements as functions of aspect and bank angles are shown in Fig. 2 (Ref. 13).

\section{B. Radar Model}

The radar model will be presented in terms of its inputs (aircraft range and RCS) and output (an estimate of the probability that an aircraft can be tracked for an interval of time).

For the sake of simplicity, assume that the radar is located at the origin of the Cartesian coordinate system $(x, y, z)$. Let $R=\sqrt{ }\left(x^{2}+y^{2}+z^{2}\right)$ be the slant range from the radar to the aircraft, that is, the aircraft range. The radar detects the aircraft by receiving a sequence of radio frequency pulses reflected from it at fixed observation times. The azimuth and range detected by the radar serve as inputs to a tracking system, typically based on one or more Kalman filters. The purpose of this tracking system is to provide a predicted aircraft position and velocity so that a decision can be made to launch a missile and guide it to intercept.

At each observation time, aircraft detection is probabilistic. ${ }^{18-21}$ A consequence of probabilistic detection is that tracking must be considered probabilistic as well. ${ }^{22-24}$ It has been shown ${ }^{27}$ that the instantaneous probability of tracking an aircraft is approximated by

$$
P_{t}=1 /\left[1+\left(c_{2} R^{4} / \sigma\right)^{c_{1}}\right]
$$

where $R$ is the slant range and $\sigma$ is the RCS. The constants $c_{1}$ and $c_{2}$ are defined by the type of radar, specifically by its power, signal processing capability, and operational settings. Based on Eq. (5), the estimated probability that the radar tracks the aircraft over an interval $[t-\Delta T, t]$ is

$$
\frac{1}{\Delta T} \int_{t-\Delta T}^{t} P_{t}(\tau) \mathrm{d} \tau
$$

\section{Missile Model}

Before missile launch, the radar must continuously track the aircraft during some response time $T_{\text {resp }}$. For example, the Russian SA-6 system is documented as having a response time of 20-22 s (Refs. 28 and 29). 
Table 1 Model parameters

\begin{tabular}{|c|c|}
\hline Parameter & Meaning \\
\hline & Aircraft \\
\hline$v$ & Speed of aircraft \\
\hline$\sigma(\lambda, \phi, u)$ & RCS function \\
\hline$U$ & Maximum allowable bank angle \\
\hline & Radar \\
\hline$c_{1}, c_{2}$ & Tracking constants \\
\hline$T_{\text {resp }}$ & Radar response time \\
\hline & Missile \\
\hline$T_{\text {fo }}$ & Missile flyout time \\
\hline
\end{tabular}

After launch, accurate missile guidance requires that the radar system maintain track on the aircraft during the time of flight of the missile. ${ }^{25}$ Assume that the launch site of the missile is collocated with the radar, and let $R$ be the aircraft range at the time the missile is fired and $v_{m}$ be the average missile speed. When it is assumed that the aircraft range does not change significantly while the missile is in flight, the missile flyout time is given by

$$
T_{\mathrm{fo}}=R / v_{m}
$$

Although $T_{\mathrm{fo}}$ depends explicitly on $R$ and $v_{m}$, for the sake of simplicity, we assume in this paper that $T_{\mathrm{fo}}$ is a constant.

If the radar system loses track of the UCAV during the response time, a missile will not be launched unless the UCAV is again tracked for an interval of $T_{\text {resp }}$. Similarly, if the radar system loses track of the UCAV during missile flyout, then another missile must be launched. Furthermore, this missile launch occurs only after the UCAV is again tracked for an interval of $T_{\text {resp }}$. Hence, for the UCAV to be shot down at time $t$, the radar system must have continuously tracked the UCAV during the interval $\left[t-\left(T_{\text {resp }}+T_{\mathrm{fo}}\right), t\right]$.

Let $P_{d}(t)$ be the probability that the UCAV will be shot down at time $t$, and define the threat window as $T \triangleq T_{\text {resp }}+T_{\text {fo }}$. Because downing of the aircraft at time $t$ requires continuous tracking over the time interval $[t-T, t]$, expression (6) yields

$$
P_{d}(t) \leq \frac{1}{T} \int_{t-T}^{t} P_{t}(\tau) \mathrm{d} \tau
$$

that is, the probability that the UCAV be downed at time $t$ is bounded from above by the probability that, just before time $t$, it be tracked over a time interval whose length is the threat window.

\section{Model Summary}

In summary, our integrated model of the aircraft-radar-missile system is as follows. The lateral acceleration $u$ determines the position of the aircraft through Eqs. (1), its aspect angle through Eqs. (2), and its bank angle through Eq. (3). The aspect, bank, and elevation angles determine the RCS through Eq. (4). The RCS and range determine the instantaneous probability of tracking through Eq. (5). Averaging the probability of tracking over the threat window through inequality (8) provides an upper bound on the probability that the UCAV is shot down.

As follows from Eqs. (1-8), the integrated model is specified by one functional and six numerical parameters listed in Table 1.

The preceding dynamic model can be made nondimensional by normalizing the aircraft speed as $v=1$ and the threat window as $T=1$.

\section{Problem Formulation}

\section{A. Mission Description}

The missions considered in this paper are to fly from a given initial location to a given destination, in a given mission time $T_{M}$, while avoiding being downed by radar-guided SAMs. Our formulation allows the specification of a sequence of waypoints that the UCAV must fly over. The heading angles of the UCAV at the waypoints and the flight times between consecutive waypoints may be free or given. Hence, the mission is specified by initial and final conditions, mission time, waypoints, and flight times between waypoints.

\section{B. Dynamic Optimization Problem}

Inequality (8) provides the motivation for the dynamic optimization problem that we pose and solve in this paper. We seek to minimize the maximum with respect to time of the right-hand side of inequality (8) subject to aircraft dynamics and boundary conditions. In other words,

$$
\min _{u} \max _{t \in\left[0, T_{M}\right]} \frac{1}{T} \int_{t-T}^{t} P_{t}(\tau) \mathrm{d} \tau
$$

subject to Eqs. (1-5) and boundary conditions, including initial conditions, final conditions, and waypoints.

Clearly, a small value of objective function (9) guarantees, as per inequality (8), a safe flight path, that is, one in which the probability that the UCAV be downed is small at all times.

\section{Optimal Control}

In this section, we use the necessary conditions of optimality for the minimax problem with moving average functional to derive qualitative properties of aircraft optimal control and characteristics of the optimal trajectory.

\section{A. Application of Necessary Condition}

The necessary conditions of optimality for minimax optimization problem (9) are derived in Appendices A and B. Here we apply these necessary conditions to problem at hand. To accomplish this, augment the state space of Eqs. (1) with

$$
\dot{\xi}(t)=\left[P_{t}(t)-P_{t}(t-T)\right] / T
$$

Then problem (9) becomes

$$
\min _{u} \max _{t \in\left[0, T_{M}\right]} \xi(t)
$$

subject to Eqs. (1-5) and boundary conditions, including initial conditions, final conditions, and waypoints.

The resulting system, with states $[x, y, \psi, \xi]^{T}$, is the same as that of Eqs. (1), with the addition of a state described by the time-delay differential equation (10). The Hamiltonian for this problem is

$$
\begin{aligned}
H= & p_{x}(t) v \cos \psi(t)+p_{y}(t) v \sin \psi(t)+p_{\psi}(t)[u(t) / v] \\
& +p_{\xi}\left\{\left[P_{t}(t)-P_{t}(t-T)\right] / T\right\}
\end{aligned}
$$

where $p_{x}, p_{y}, p_{\psi}$, and $p_{\xi}$ are the costates.

We now consider the case in which the maximum occurs at a finite number of isolated points $t_{1}, t_{2}, \ldots, t_{k}$, although similar arguments apply, with minor changes, to the case that the minimax occurs over finite intervals. As shown in Appendices A and B for the case of isolated maxima, the necessary conditions of optimality define two different optimization problems. Each will be addressed in turn.

\section{B. Optimization Problem 1}

Let $t_{i}$ be the time at which $\xi(t)$ achieves a maximum. We refer to the optimization problem during the interval $\left[t_{i}-T, t_{i}\right]$ as optimization problem 1 . For this problem, the costate equations become

$$
\begin{gathered}
\dot{p}_{x}=\frac{\mu_{i}}{T} \frac{\partial P_{t}(t)}{\partial x}, \quad \dot{p}_{y}=\frac{\mu_{i}}{T} \frac{\partial P_{t}(t)}{\partial y} \\
\dot{p}_{\psi}=p_{x}(t) v \sin \psi(t)-p_{y}(t) v \cos \psi(t)+\frac{\mu_{i}}{T} \frac{\partial P_{t}(t)}{\partial \psi}, \quad \dot{p}_{\xi}=0
\end{gathered}
$$

and when $\partial P_{t} / \partial u \neq 0$, the optimal control is found as the solution to

$$
\frac{p_{\psi}}{v}-\frac{\mu_{i}}{T} \frac{\partial P_{t}}{\partial u}=0
$$

where $\mu_{i}>0$ and

$$
\sum_{i=1}^{k} \mu_{i}=1
$$


When $\partial P_{t} / \partial u=0$, the optimal control is found by maximizing $H$, that is, by choosing

$$
u=U \operatorname{sign}\left(p_{\psi}\right)
$$

In addition, a singular solution is possible when $p_{\psi}=0$ and $\dot{p}_{\psi}=0$. This occurs when

$$
p_{x}(t) v \sin \psi(t)-p_{y}(t) v \cos \psi(t)+\frac{\mu_{i}}{T} \frac{\partial P_{t}(t)}{\partial \psi}=0
$$

As an example, if the current state $[x, y, \psi, \xi]^{T}$ results in $\partial P_{t}(t) / \partial \psi=0$ and if

$$
\tan \psi=p_{y} / p_{x}
$$

then the condition for a singular solution is satisfied. Thus, when $\partial P_{t} / \partial u=0$, the optimum control is either bang-bang, as given by Eq. (15), or bang-singular-bang when a singular condition exists, as in Eq. (16).$^{30}$ On the other hand, if the current state results in $\partial P_{t}(t) / \partial u \neq 0$, the optimal control is defined by Eq. (14).

We will say that when the necessary conditions of optimization problem 1 apply, the aircraft is under threat.

\section{Optimization Problem 2}

Let $\left[t_{j}, t_{j}^{\prime}\right]$ be an interval that is disjoint from any interval $\left[t_{i}-T, t_{i}\right]$ described in optimization problem 1 . We refer to the optimization problem during the interval $\left[t_{j}, t_{j}^{\prime}\right]$ as optimization problem 2. During any such interval, the costate equations are

$$
\begin{gathered}
\dot{p}_{x}=0, \quad \dot{p}_{y}=0 \\
\dot{p}_{\psi}=p_{x}(t) v \sin \psi(t)-p_{y}(t) v \cos \psi(t), \quad \dot{p}_{\xi}=0
\end{gathered}
$$

The optimal control is either bang-bang as in Eq. (15), or singular if $p_{\psi}=0$ and

$$
p_{x}(t) v \sin \psi(t)-p_{y}(t) v \cos \psi(t)=0
$$

However, this equation is just equivalent to

$$
\tan \psi=p_{y} / p_{x}
$$

Thus, during any interval $\left[t_{j}, t_{j}^{\prime}\right]$ described in this optimization problem, the optimal control is always bang-bang or bang-singularbang.

We will say that when the necessary conditions of optimization problem 2 apply, the aircraft is not under threat.

\section{Properties of Optimal Paths}

The exact solution of problem (9) requires that the times $t_{i}$ when the isolated maxima occur, as well as the boundary conditions at the beginning and end of each interval $\left[t_{i}-T, t_{i}\right]$, be selected optimally.

Unfortunately, the necessary conditions do not indicate how these times or boundary conditions are to be chosen. Thus, the necessary conditions of optimality do not define a unique candidate for the solution of problem (9). Additionally, an exact solution requires knowledge of the aircraft RCS because $P_{t}$ depends on this function. In spite of these limitations, it is possible to characterize the qualitative properties of optimal controls for the two optimization problems and use these properties to develop an efficient numerical optimization method.

\section{A. Qualitative Properties for Optimization Problem 1:} Aircraft Under Threat

The purpose of this subsection is to show that when the aircraft is under threat, the optimal control is practically bang-bang. When optimization problem 1 applies, consider the following assumptions, which simplify the problem enough to determine the qualitative nature of the trajectory.

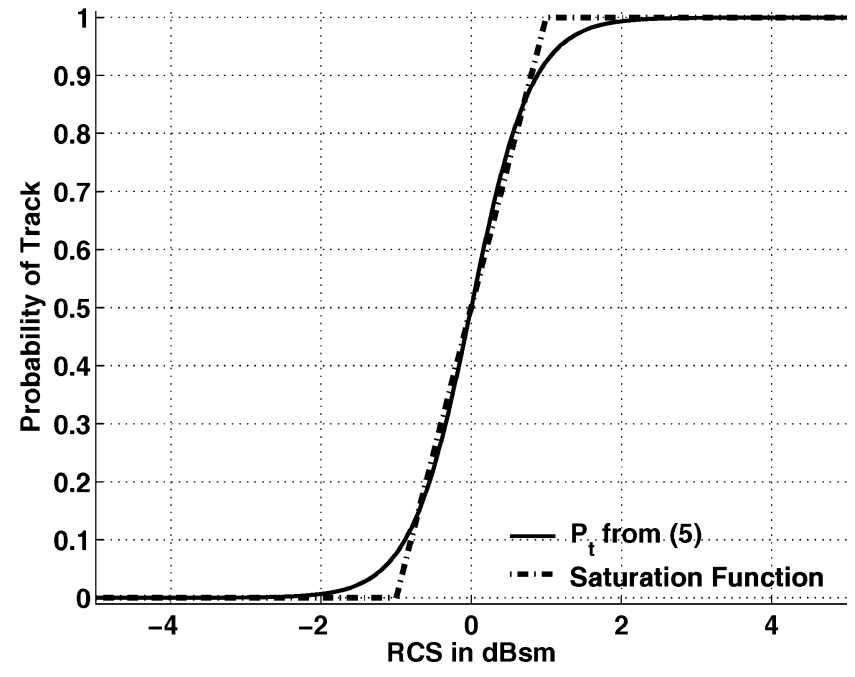

Fig. $3 P_{t}$ vs RCS.

1) Assume $\partial P_{t} / \partial u=0$ from the nose or tail aspect.

2) Assume from any aspect angle other than the nose or tail the aircraft RCS increases monotonically as the magnitude of bank angle increases. Additionally, $\partial \sigma / \partial \mu=0$ at $\mu=0$.

3) Assume $P_{t}$ is a saturation function of RCS. [See Fig. (3) where the saturation function and the exact expression (5) are shown.] The effect of this approximation is to set $\partial P_{t} / \partial \sigma \equiv 0$, hence, $\partial P_{t} / \partial u \equiv 0$, for all but a small range of values of $\sigma$.

Under these conditions, the optimal control necessarily has the following properties:

1) If $p_{\psi} \neq 0$ when flying directly toward or away from a radar, then a turn must be initiated using maximum bank. Indeed, because $\partial P_{t} / \partial u=0$, and $p_{\psi} \neq 0$, the control is bang-bang by Eq. (15)

2) If $p_{\psi} \neq 0$ and $P_{t}=1$ when flying straight, then a turn must be initiated using maximum bank. Indeed, the fact that $P_{t}=1$ during straight flight indicates $\partial P_{t} / \partial u=0$ for all $\mu$. Because $p_{\psi} \neq 0$, the control is bang-bang by Eq. (15).

3) When $p_{\psi}=0$, no turn is required. Indeed, if $p_{\psi}=0$ then $\partial P_{t} / \partial u=0$ by Eq. (14). Two conditions that satisfy this requirement are when $\mu=0$ or when $P_{t}$, as a function of RCS, is saturated. In either case, $\mu=0$ satisfies the necessary conditions.

\section{B. Qualitative Properties for Optimization Problem 2: Aircraft Not Under Threat}

The purpose of this subsection is to show that when the aircraft is not under threat, the optimal trajectory may be chosen as a sequence of maximum rate turns connected by intervals of straight flight. When the aircraft is not under threat, the necessary conditions are given by Eqs. (18). They are equivalent to the optimal control problem that results from setting $P_{t}=0$ in the Hamiltonian (12). When the same reasoning as earlier is used the control is either bang-bang or bang-singular-bang with singular control occurring when

$$
\tan \psi=p_{y} / p_{x}
$$

Because, in this case, the cost functional is independent of the control input, there is no unique solution. However, we choose to treat this as a minimum-time problem because this results in the minimum path length, which has the same necessary conditions. Waypoints are treated as interior-point state constraints that necessitate jump discontinuities in $p_{x}, p_{y}$, and $p_{\psi}$ (Ref. 30).

One solution to such a problem is to fly straight from one waypoint to the next, turning with the maximum possible bank after overflying the current point. While flying straight, we meet the condition $\tan \psi=p_{y} / p_{x}$ and choose $u=0$. When overflying a waypoint, jump discontinuities in $p_{x}, p_{y}$, and $p_{\psi}$ necessitate the use of maximum bank until heading toward the next point and again meeting the condition for straight flight. 


\section{Numerical Procedure}

The discussion in Sec. V suggests that a reasonable suboptimal trajectory might be achieved by using only zero bank or maximum bank. As a result, our numerical method for solving the dynamic optimization problem (9) is as follows. The optimization is assumed to occur over the interval $\left[0, T_{M}\right]$. An initial path is chosen by specifying a series of waypoints and control points. We distinguish between these two by requiring that waypoints be fixed, whereas control points are allowed to move as required for optimality.

The path is determined by requiring overflight of each point, in turn. Overflight of control points is followed by a maximum bank turn to roll out on a heading toward the next point. Turns at waypoints are not required. We then treat the coordinates of the control points as inputs to a finite dimensional, nonlinear programming problem, which we solve numerically using the MATLAB ${ }^{\circledR}$ Optimization Toolbox function fminimax.

In our experience, the optimization problem (9) typically possesses several local minima, and the particular minimum to which the algorithm converges depends on the initial choice of control points. A good initial choice of these control points requires heuristic expertise, which can be codified in a variety of forms, such as rule-based or table look-up. The development of this initialization module is a subject of future work. For the examples that follow, convergence typically occurred within 35 iterations, with each iteration requiring approximately $2 \mathrm{~s}$ on a $300-\mathrm{MHz}$, Pentium II personal computer.

\section{Numerical Optimization Results}

\section{A. RCS Example}

A simplified example of an RCS model for an aircraft is that of an ellipsoid. Although this model does not represent the RCS of a particular aircraft, it captures three important characteristics: 1) relatively small frontal RCS, 2) larger beam aspect RCS, and 3) relatively large RCS when viewed from above or below.

Following Ref. 18, the RCS of an ellipsoid is given by

$$
\begin{gathered}
\sigma(\lambda, \phi, \mu)=\frac{\pi a^{2} b^{2} c^{2}}{\left(a^{2} \sin ^{2} \lambda_{e} \cos ^{2} \mu_{e}+b^{2} \sin ^{2} \lambda_{e} \sin ^{2} \mu_{e}+c^{2} \cos ^{2} \lambda_{e}\right)^{2}} \\
\lambda_{e}=\arccos [\cos (\phi) \cos (\lambda)] \\
\mu_{e}=\mu-\arctan \left[\frac{\tan (\phi)}{\sin (\lambda)}\right]
\end{gathered}
$$

Future research will consider other models that may better approximate actual aircraft RCS. Note, however, that actual aircraft RCSs are often classified.

\section{B. Common Parameters}

The aircraft model assumes that the UCAV has a constant speed of $v=252 \mathrm{~m} / \mathrm{s}(0.8 M)$ and its RCS, in Fig. 4 , is characterized by the parameters $(a, b, c)=(0.3172,0.1784,1.003)$. The maximum bank angle is $U=78.5 \mathrm{deg}$ and corresponds to a 5- $g$ level turn. The radar model assumes that all radars have a response time $T_{\text {resp }}=30 \mathrm{~s}$ and a fixed missile flyout time $T_{\mathrm{fo}}=30 \mathrm{~s}$. Hence, the threat window of the radar-missile subsystems is $1 \mathrm{~min}$. Numeric calculation will use the approximation for $P_{t}$ in Eq. (5). The aircraft altitude is set to $z=15.1 \mathrm{~km}$ and the mission time $T_{M}$ is assumed to be $750 \mathrm{~s}$.

\section{Scenario 1}

\section{Mission Specifications}

This mission is specified in terms of the radar position, initial UCAV position and velocity vector, waypoints, destination, and the arrival time at each position. Here, and in subsequent scenarios, the distance is expressed in kilometers, time is in seconds, and speed is in meters per second. Specifically, we assume radar position $=(0,0)$; initial position $\mathrm{A}=(-100,-12)$, $t=0$; initial velocity $\left(v_{x}, v_{y}\right)=(252,0)$; waypoint $\mathrm{B}=(-74.1,0)$,

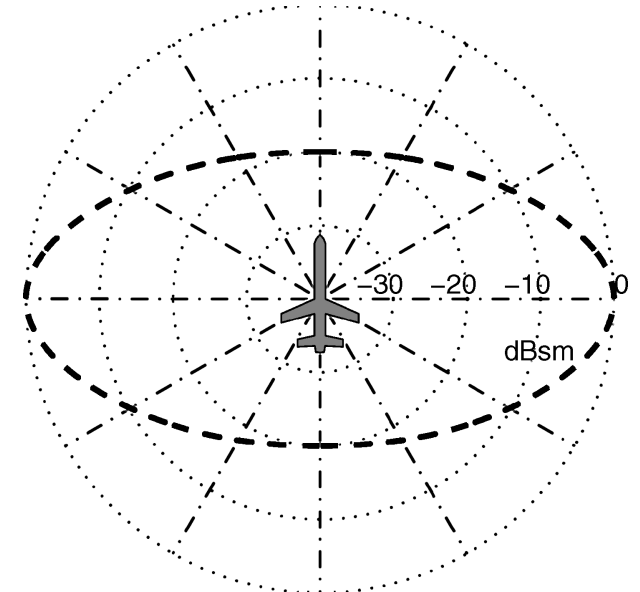

a) UAV RCS as function of aspect angle

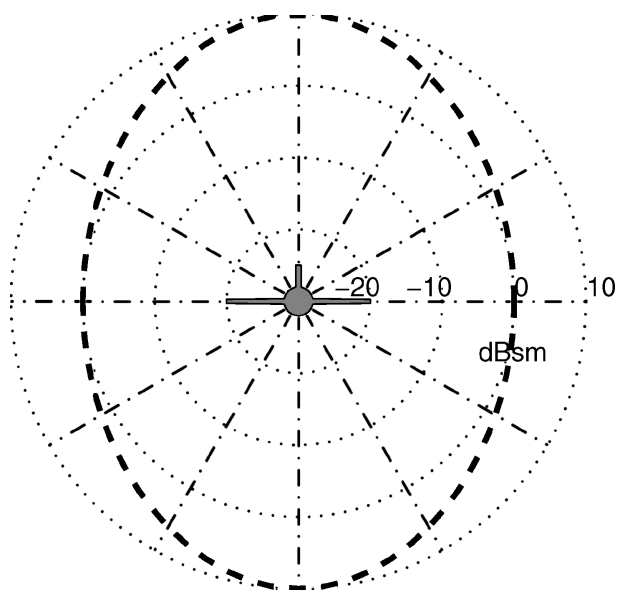

b) UAV RCS as function of bank angle

Fig. 4 Dependence of UCAV RCS on aspect and bank angles.

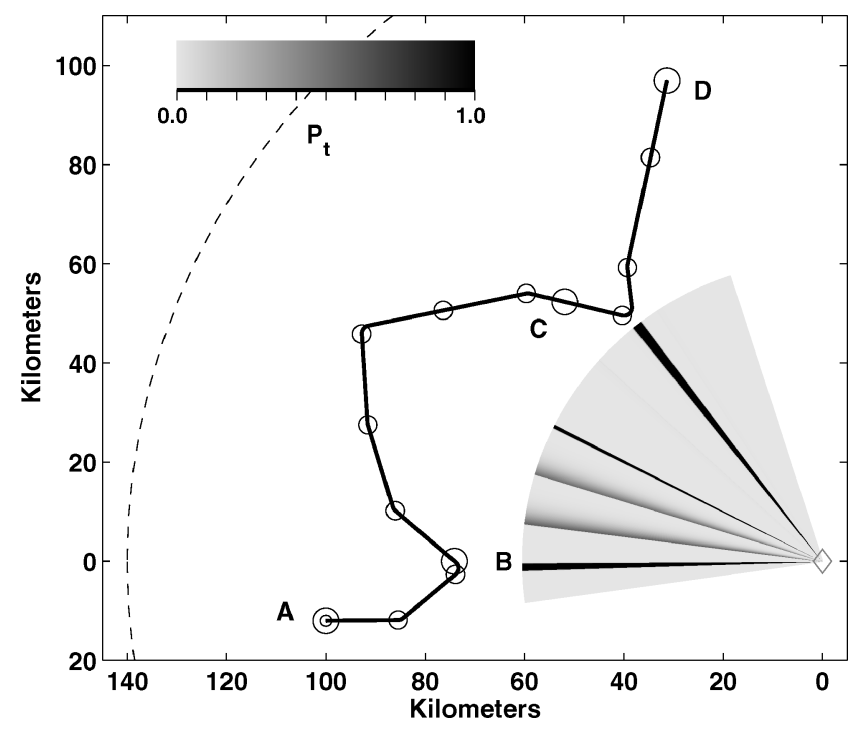

Fig. 5 Optimal path based on model (1-8).

$t=135.0$; waypoint $\mathrm{C}=(-51.9,52.3), t=522.0$; and destination $\mathrm{D}=(-31.3,96.9), t=750.0$.

\section{Optimal Path}

Using these mission specifications and the optimization approach outlined in Sec. VI, we have calculated the optimal path shown in Fig. 5. In Fig. 5 and subsequently the following conventions are used. 
1) Initial position, waypoints, and destination are alphabetically labeled large circles, whereas control points are small circles. Under some circumstances, a turn is required at a waypoint, that is, we treat the waypoint as a fixed control point. In this case, concentric large and small circles are used.

2) Radar position is shown by a diamond.

3) The range at which $P_{t}=0.5$, for a target with $\operatorname{RCS} \sigma=1 \mathrm{~m}^{2}$, is scenario dependent and is shown by a dashed arc of circle.

4) The UCAV ground track is shown by a solid line.

5) The instantaneous $P_{t}$ is indicated by the darkness of a line of sight from the radar toward the aircraft as shown in the legends.

On the optimal path, the maximum value of inequality (8) is 0.159 , confirming its safety. The optimal path, shown in Fig. 5, corroborates the tactical recommendation given to fighter pilots facing SAM threats: "deny range, aspect, and aim." ${ }^{26}$ Indeed, consider the BC leg: Range is denied by flying as far from the radar as the time constraint allows; aspect is denied by avoiding, as much as possible, to show the larger RCS beam aspect; aim is denied by making the periods of time, during which the UCAV is continuously tracked, short.

This scenario exhibits a characteristic property of optimal paths for aircraft with non-uniform RCS under threat: The optimal trajectory exploits weaving maneuvers to avoid long continuous exposure of aspects with large RCS. This property is observed in all scenarios considered to date.

\section{Scenario 2}

\section{Multiple-Radar Performance Index}

When multiple radars are present, the optimization problem (9) must be modified. If there are two radars, and they operate independently, then the UCAV must minimize the maximum value of the integral in problem (9) for each radar at each instant of time. Let $i \in\{1,2\}$ represent the enumeration of the radars. Then the optimization problem is

$$
\min _{u} \max _{t \in\left[0, T_{M}\right]} \max _{i \in\{1,2\}} \frac{1}{T} \int_{t-T}^{t} P_{t i}(\tau) \mathrm{d} \tau
$$

subject to Eqs. (1-5) and boundary conditions, including initial conditions, final conditions, and waypoints, where $P_{t i}$ is the probability that the UCAV is tracked by radar $i$.

\section{Mission Specifications}

Radar 1 is located at $(0,25)$ and radar 2 is located at $(0,-25)$, that is, the separation is $50 \mathrm{~km}$. The initial position $\mathrm{A}=(-55,0), t=0$; destination $\mathrm{B}=(55,0), t \leq 750$; and flight path must pass between the radars.

\section{Optimal Path}

An optimal path for this mission is in Fig. 6. The maximum value of inequality (8) is 0.0948 for both radars 1 and 2 . The flight time between $\mathrm{A}$ and $\mathrm{B}$ is $477.0 \mathrm{~s}$.

\section{Comparison with the Literature}

One recommended method for selecting a path between radars is to maximize the minimum distance to each of them ${ }^{4-7}$. The result is a path that follows the boundaries of a Voronoi diagram, that is, it is equidistant from the closest radars. Our comparison is in Fig. 7 and meets the specifications for scenario 2 . The recommended path is the perpendicular bisector of the line connecting the two radars.

Under the assumptions of our model, this recommended path results in a high probability that the UCAV is downed. As can be seen in Fig. 7, the instantaneous value of $P_{t}$ remains high for quite a long time. This results in a high value for the right-hand side of inequality (8), which is identical for both radars and equals 0.9644 . The danger of this path is due to the long exposure of a relatively large RCS to both radars.

Our model accounts for the combined effects of nonisotropic aircraft RCS, the coupling between aircraft dynamics and the RCS presented to each radar the radars' tracking capabilities, and the

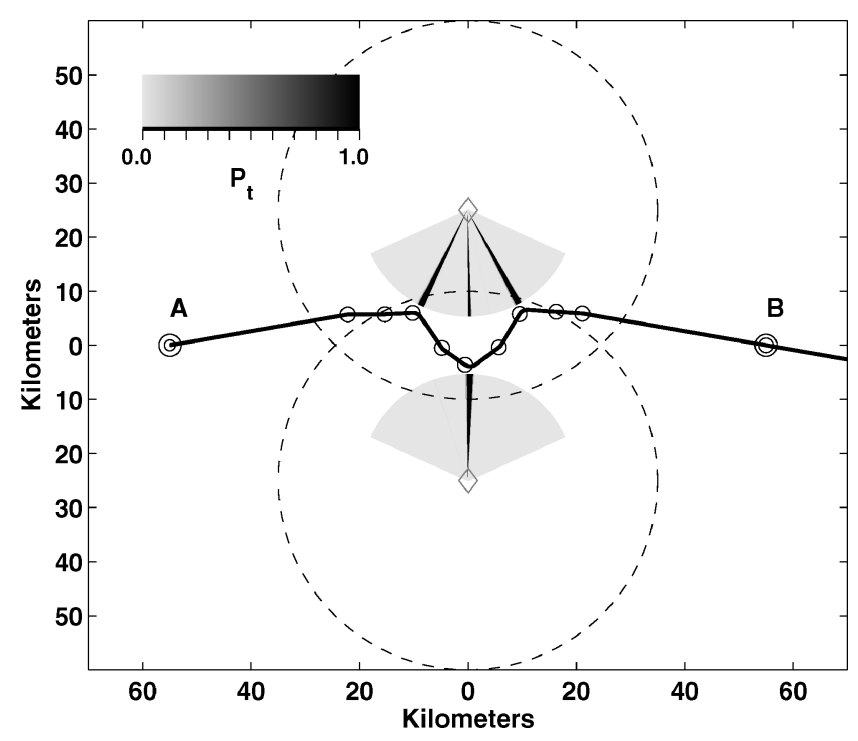

Fig. 6 Optimal path between two radars separated by 50 km.

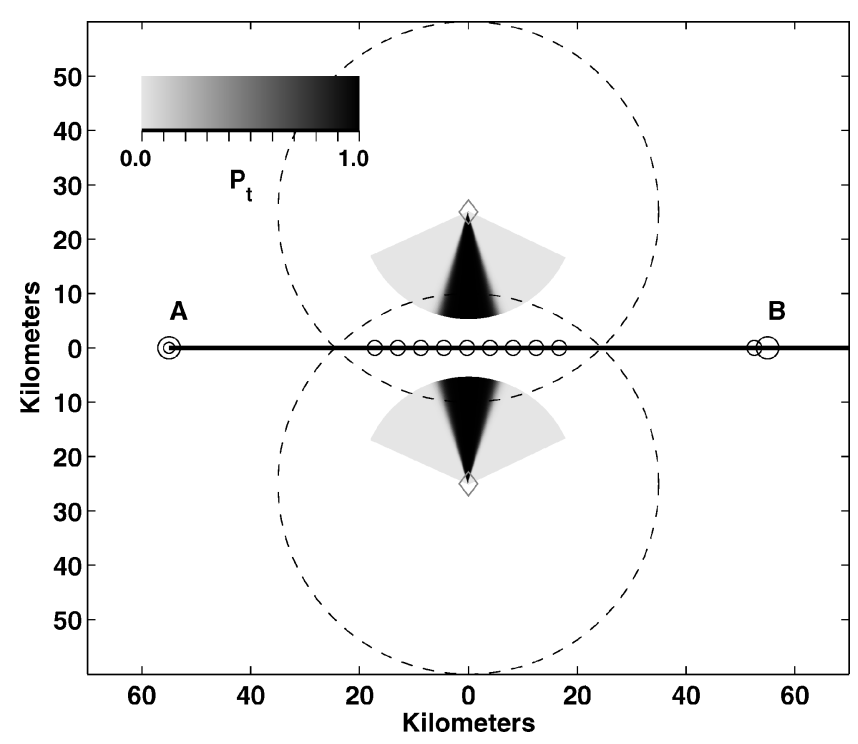

Fig. 7 Flight path based on Refs. 4-7.

requirement for the radars to maintain tracking before missile launch and during missile flyout. In our experience, any failure to account for these effects yields paths that may lead to long exposures of relatively large RCS, thereby being unacceptably dangerous.

\section{E. Scenario 3}

1. Motivation and Specifications

The preceding two scenarios assumed that the location of each threat was known at the time the path was planned. We now consider the case where the location or even the existence of a threat cannot be determined during mission planning, but this information becomes available during the mission.

Our scenario assumes two radars and that the existence and location of one of them are known. The solution to problem (9) yields an optimal path considering this single radar threat. During the mission, however, the other radar is detected. The problem then becomes one of optimizing the path from the point of detection forward to the destination, taking into account this new pop-up threat, that is, solving problem (25). The critical question to be answered is whether the mission can still be safely accomplished, satisfying all constraints. A secondary question is whether relaxing a constraint, for example, allowing the UCAV to bypass a waypoint or arrive at the destination late, would allow the mission to be completed on a safer path. Most 
important, these questions must be answered, and the new flight path must be determined, quickly. These questions will be considered in two separate cases.

Formally, the specifications for this scenario are as follows. The pop-up threat, radar 1 , is located at $(0,25)$ and is detected at $t=146.5$; radar 2, known at the time of mission planning, is located at $(0,-25)$; initial position $\mathrm{A}=(-55,0), t=0$; and destination $\mathrm{B}=(55,0), 146.5<t \leq 750$.

\section{Optimal Path Based on a Single Radar}

An optimal path, considering only radar 2, is shown in Fig. 8. It is safe in that the optimization of problem (9) yields a value of 0.0082 . The flight time from A to B is $566.7 \mathrm{~s}$.

\section{Case 1}

In this case, we consider the arrival time at the destination fixed at the value determined by the initial optimal path. A new path, optimized to account for radar 1, is shown in Fig. 9. The optimization routine need only replan the path between the point of detection and the destination. This new path is not as safe as the original path, with optimization of problem (25) yielding 0.6025. Furthermore, replanning required selection of a new initial path for the optimization routine.

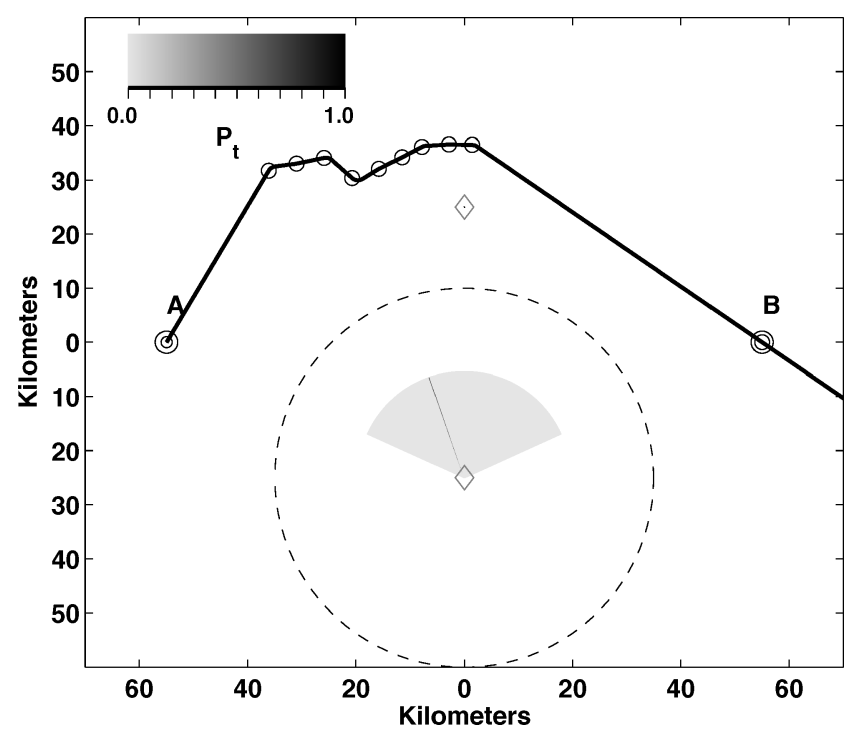

Fig. 8 Initial optimal path.

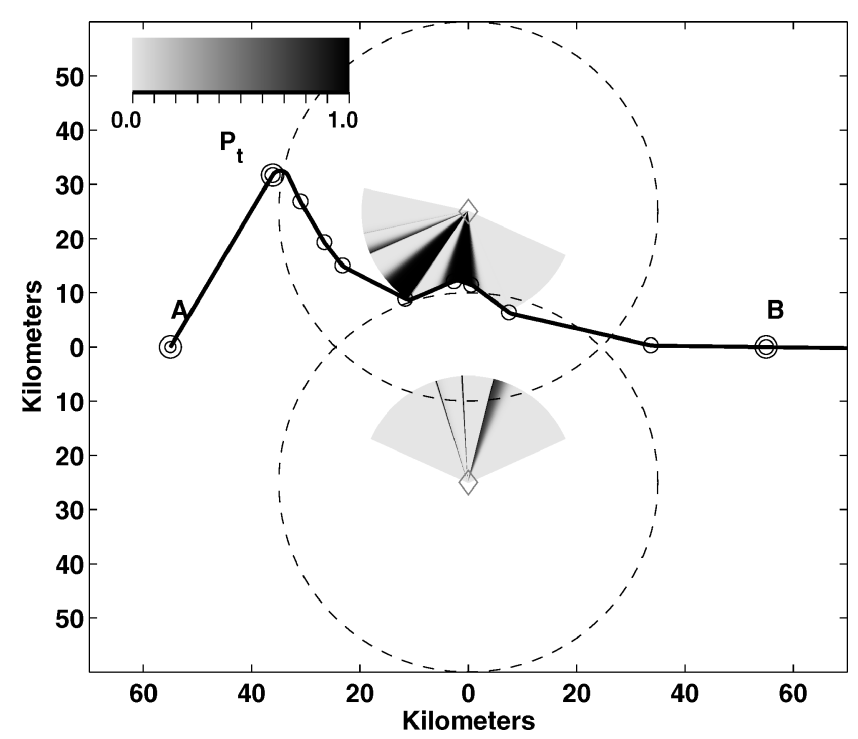

Fig. 9 Replanned path with timing constraint.

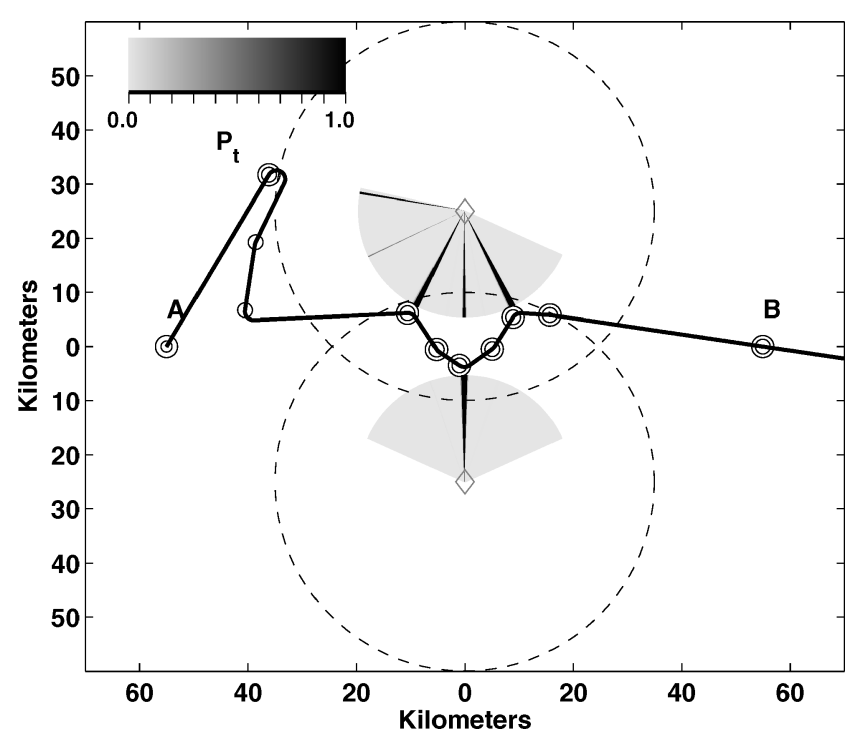

Fig. 10 Initial optimal path and replanned path with no timing constraint.

In the case of an actual pop-up threat, the selection of a new path and the subsequent replanning process may require more time than is available inflight while the UCAV is subject to the threat of radar 1. Therefore, we consider another case with free arrival time.

\section{Case 2}

In this case, we assume that the arrival time at the destination is not fixed. We would like to know whether a path can be found that is safer than the new path shown in Fig. 9. Additionally, we would like to know this answer quickly. We know, from scenario 2 , that the optimal path between two radars separated by $50 \mathrm{~km}$ is as shown in Fig. 6. This path information could be stored onboard the UCAV. When radar 1 is detected, replanning would be based on the stored information. The optimization routine need only find a safe path from the point when radar 1 is detected to any point on the stored path. Because this inflight optimization is done over a relatively short connecting path consisting of two control points, this can be done more quickly. Such a resulting replanned path is shown in Fig. 10. Notice that the stored optimal path originally shown in Fig. 6 is now shown as a series of waypoints where turns are required (concentric circles). These points cannot be changed by the optimization algorithm. The two control points subject to optimization are the ones depicted by small circles.

In this example, the flight time from A to B on the new path is $686.1 \mathrm{~s}$, or $119.4 \mathrm{~s}$ longer than before. However, only two control points needed to be optimized. The time required to replan was reduced by a factor of 10 . Additionally, this new path is much safer than that of the earlier example, with optimization of problem (25) yielding 0.0948 as it was in scenario 2 .

Case 2 demonstrates another method for inflight replanning that is more responsive to changes in the threat. Because we used stored information from scenario 2 as an initial path, we were able to replan a new path much more quickly than if we had to consider the entire path to the destination. This suggests that a scheme of precalculating and storing the optimal paths for a variety of conditions, then recalling the information when needed, may allow quicker flight planning than recalculating the entire optimal path for each change in the threat.

\section{Conclusions}

We have presented a new, integrated model for optimal path planning for UCAVs in the presence of radar-guided missile threats. This model accounts for the combined effects of nonisotropic aircraft RCS, the coupling between aircraft dynamics and the RCS presented to a radar, the radar's tracking capability, and the requirement for the radar to maintain tracking before missile launch and 
during missile flyout. The danger to the UCAV for a given path is quantified as an upper bound on the probability that the radar is able to track the UCAV long enough to guide a missile to intercept. On the basis of this model, a major conclusion of the present study is that an accurate quantification of the danger to the UCAV indeed requires accounting for the combination of the effects outlined.

Our analysis has also shed light on properties of optimal paths, that is, paths that minimize the danger to the UCAV. These properties have been shown to enable discretized approximation of the paths and to facilitate efficient numerical optimization.

Most important, this paper provides a scientific basis for the flying tactics inculcated to combat pilots. Indeed, optimal flight paths deny range, aspect, and aim by avoiding long exposures of aspects with large RCS.

\section{Appendix A: Summary of Results Derived in Reference 31}

We begin by summarizing in Appendix A the necessary conditions of optimality for the minimax optimal control problem as developed in Ref. 31 and applied in Refs. 32-35. Results are then extended in Appendix B to the moving average functional used in this paper.

Let $F[x(t)]$ be a function depending on the states $x$ of a dynamic system

$$
\dot{x}=\boldsymbol{f}(\boldsymbol{x}, u)
$$

where $u$ is a scalar control defined over a time interval $\left[t_{0}, t_{f}\right]$. The boundary conditions are

$$
\begin{gathered}
x\left(t_{0}\right)=x_{0} \\
\omega_{f}\left[x\left(t_{f}\right)\right]=0
\end{gathered}
$$

where $t_{f}$ can be given implicitly by Eq. (28) or explicitly. The minimax optimal control problem is stated as

$$
\min _{u} \max _{t \in\left[t_{0}, t_{f}\right]} F[x(t)]
$$

Conditions for existence of a solution to problem (A4) are well known in the literature ${ }^{36}$ and will not be restated here. Additionally, we recognize, as has been documented, ${ }^{31}$ that the minimax solution is generally not unique.

The approach in Ref. 31 is to augment the states of the system with

$$
\dot{x}_{n+1}=0
$$

then minimize the constant $x_{n+1}$ subject to the inequality constraint

$$
F[\boldsymbol{x}(t)]-x_{n+1} \leq 0, \quad t_{0} \leq t \leq t_{f}
$$

Note that minimizing $x_{n+1}$ and satisfying the constraint (A6) account for the minimization and maximization in problem (A4). This approach transforms the minimax optimization problem (A4) into a Mayer-type optimal control problem subject to the inequality constraint (A6), for which necessary conditions are readily available (see Ref. 37). The Hamiltonian for such a problem is

$$
\begin{aligned}
H(\boldsymbol{x}, \boldsymbol{p}, u) & =\boldsymbol{p}^{T} \boldsymbol{f}(\boldsymbol{x}, u)+p_{n+1} f_{n+1}(\boldsymbol{x}, u) \\
& =\boldsymbol{p}^{T} \boldsymbol{f}(\boldsymbol{x}, u)
\end{aligned}
$$

because $f_{n+1}(\boldsymbol{x}, u)=0$ by Eq. (30).

The necessary conditions of optimality require the consideration of two cases. In the first case, the maximum value of $F(x)$ occurs at a finite number of isolated points, whereas in the second, it occurs over some finite intervals, that is, a flat maximum. In other words, this means that, in the first case, equality (A6) is achieved at a set of isolated points, whereas, in the second case, equality is achieved on a set of intervals. A solution may have instances of both cases. The necessary conditions differ for these two cases, and it must first be determined which case takes place before the conditions can be applied.
For the first case, suppose that, for an optimal pair $u(t)$ and $\boldsymbol{x}(t)$, $t_{0} \leq t \leq t_{f}, F[\boldsymbol{x}(t)]$ attains its maximum $k$ times at isolated points $t_{1}, t_{2}, \ldots, t_{k}$. Then, according to Ref. 31, there exists an adjoint vector $\boldsymbol{p}(t)$ such that the following conditions are satisfied:

$$
\begin{gathered}
\dot{\boldsymbol{p}}(t)=-\frac{\partial H}{\partial \boldsymbol{x}}, \quad t \in\left[t_{0}, t_{1}\right)\left(t_{1}, t_{2}\right), \ldots,\left(t_{k}, t_{f}\right] \\
H(\boldsymbol{x}, \boldsymbol{p}, u)=\max _{v \in U} H(\boldsymbol{x}, \boldsymbol{p}, v)=C \\
\boldsymbol{p}\left(t_{i}^{+}\right)=\boldsymbol{p}\left(t_{i}^{-}\right)+\mu_{i}\left[\frac{\partial F(\boldsymbol{x})}{\partial \boldsymbol{x}}\right] \\
p_{n+1}\left(t_{i}^{+}\right)=p_{n+1}\left(t_{i}^{-}\right)-\mu_{i} \\
\sum_{i=1}^{k} \mu_{i}=1 \\
\mu_{i}>0, \quad i=1,2, \ldots, k
\end{gathered}
$$

where $C$ in Eq. (A9) is a constant and is equal to zero if $t_{f}$ is unspecified.

For the second case, suppose $F(\boldsymbol{x})$ attains its maximum value over the intervals $\left(t_{1}, t_{1}^{\prime}\right), \ldots,\left(t_{k}, t_{k}^{\prime}\right)$. Assume that the so-called regularity condition is met, that is,

$$
\frac{\partial \dot{F}(\boldsymbol{x}, u)}{\partial u} \neq 0, \quad \forall t \in\left(t_{i}, t_{i}^{\prime}\right), i=1, \ldots, k
$$

Then, in accordance with Ref. 31, outside the intervals $\left(t_{1}, t_{1}^{\prime}\right), \ldots,\left(t_{k}, t_{k}^{\prime}\right)$, Eqs. (A8) and (A9) apply. During the intervals $\left(t_{i}, t_{i}^{\prime}\right)$, the necessary conditions of optimality are

$$
\begin{gathered}
\dot{\boldsymbol{p}}(t)=\frac{-\partial H}{\partial \boldsymbol{x}}+v_{i}\left[\frac{\partial \dot{F}(\boldsymbol{x}, u)}{\partial \boldsymbol{x}}\right] \\
H(\boldsymbol{x}, \boldsymbol{p}, u)=\max _{v \in \omega_{i}(x)} H(\boldsymbol{x}, \boldsymbol{p}, v)=C
\end{gathered}
$$

where $v_{i}(t)$ is a scalar function satisfying

$$
\frac{\partial H(\boldsymbol{x}(t), \boldsymbol{p}(t), u(t))}{\partial u}=v_{i}(t)\left[\frac{\partial \dot{F}(\boldsymbol{x}(t), u(t))}{\partial u}\right]
$$

Furthermore, the jump conditions given by Eqs. (A10-A13) remain applicable.

\section{Appendix B: Extension to Moving Average Functional}

The minimax optimal control problem with moving average functional is stated as

$$
\min _{u} \max _{t \in\left[t_{0}, t_{f}\right]} \frac{1}{T} \int_{t-T}^{t} \rho[x(\tau), u(\tau)] \mathrm{d} \tau
$$

where $\boldsymbol{x}(t), u(t)$, and $\left[t_{0}, t_{f}\right]$ are as in Eqs. (A1-A4), $\rho[\boldsymbol{x}(t), u(t)] \geq$ 0 is a cost function, and $T$ is the time interval over which the moving average is considered.

To obtain the necessary conditions, we augment the state space with two new components,

$$
\begin{gathered}
\dot{x}_{n+1}=\rho\{[x(t), u(t)]-\rho[x(t-T), u(t-T)]\} / T \\
\dot{x}_{n+2}=0
\end{gathered}
$$

Clearly,

$$
x_{n+1}=\frac{1}{T} \int_{t-T}^{t} \rho[x(\tau), u(\tau)] \mathrm{d} \tau
$$


and the optimization problem becomes

$$
\min _{u} \max _{t \in\left[t_{0}, t_{f}\right]} x_{n+1}
$$

Analogously to the method in Appendix A, we minimize $x_{n+2}$ subject to the inequality constraint

$$
x_{n+1}-x_{n+2} \leq 0
$$

Our system differs from that of Appendix A because we include the time-delay differential equation (B2).

In summary, the resulting Mayer-type optimal control problem is as follows:

$$
\min x_{n+2}
$$

subject to

$$
\begin{gathered}
\dot{\boldsymbol{x}}=\boldsymbol{f}(\boldsymbol{x}, u) \\
\dot{x}_{n+1}=\{\rho[\boldsymbol{x}(t), u(t)]-\rho[\boldsymbol{x}(t-T), u(t-T)]\} / T \\
\dot{x}_{n+2}=0 \\
u(t) \in U
\end{gathered}
$$

and the inequality constraint

$$
x_{n+1}-x_{n+2} \leq 0
$$

with boundary conditions

$$
\begin{gathered}
\boldsymbol{x}\left(t_{0}\right)=\boldsymbol{x}_{0} \\
\omega_{f}\left(\boldsymbol{x}_{f}\right)=0 \\
\rho[\boldsymbol{x}(\tau), u(\tau)] \text { given, } \quad-T \leq \tau \leq 0 \\
x_{n+1}\left(t_{0}\right)=\frac{1}{T} \int_{-T}^{0} \rho[\boldsymbol{x}(\tau), u(\tau)] \mathrm{d} \tau \\
x_{n+2}\left(t_{0}\right) \text { free }
\end{gathered}
$$

In general, the necessary conditions for this problem have been derived in Ref. 38. However, to obtain these conditions in a form more convenient for the current study, we rederive them.

Introduce the Hamiltonian,

$$
H=\boldsymbol{p}^{T} \boldsymbol{f}(\boldsymbol{x}, u)+p_{n+1}(\{\rho[\boldsymbol{x}(t), u(t)]-\rho[\boldsymbol{x}(t-T), u(t-T)]\} / T)
$$

When $x_{n+1}-x_{n+2}<0$, standard results of optimal control with time delay ${ }^{37,39}$ give

$$
\begin{gathered}
\dot{\boldsymbol{x}}=\frac{\partial H}{\partial \boldsymbol{p}} \\
\dot{x}_{n+1}=\{\rho[\boldsymbol{x}(t), u(t)]-\rho[\boldsymbol{x}(t-T), u(t-T)]\} / T \\
\dot{x}_{n+2}=0 \\
\dot{\boldsymbol{p}}=-\frac{\partial}{\partial \boldsymbol{x}} H(\cdot)-\left.\frac{\partial}{\partial \boldsymbol{x}_{d}} H(\cdot, s)\right|_{s=t+T}, \quad t_{0}<t<t_{f}-T \\
=-\frac{\partial}{\partial \boldsymbol{x}} H(\cdot), \quad t_{f}-T<t<t_{f} \\
\dot{p}_{n+1}=0 \\
\dot{p}_{n+2}=0
\end{gathered}
$$

where the subscripted variable $\boldsymbol{x}_{d}$ indicates differentiation with respect to the delayed states. It is possible to rewrite the expression for the costate dynamics, Eqs. (B22), in the form

$$
\begin{array}{r}
\dot{\boldsymbol{p}}=-\frac{\partial\left(\boldsymbol{p}^{T} \boldsymbol{f}(\boldsymbol{x}, u)\right)}{\partial \boldsymbol{x}}+\frac{\partial \rho(\boldsymbol{x}(t), u(t))}{\partial \boldsymbol{x}} \frac{\left[p_{n+1}(t+T)-p_{n+1}(t)\right]}{T} \\
t_{0}<t<t_{f}-T \text { (B26) }
\end{array}
$$

The optimal control $u(t)$ maximizes $H$ and is found as the solution to

$$
\frac{\partial\left[\boldsymbol{p}^{T} \boldsymbol{f}(\boldsymbol{x}, u)\right]}{\partial \boldsymbol{u}}=\frac{\partial \rho[\boldsymbol{x}(t), u(t)]}{\partial \boldsymbol{u}} \frac{\left[p_{n+1}(t+T)-p_{n+1}(t)\right]}{T}
$$

when the indicated partial derivatives are nonzero. Thus, the state and costate dynamics are given by Eqs. (B19-B26) whereas the optimum control is given by Eq. (B27).

Consider now the first of the two cases presented in Appendix A, that is, when $x_{n+1}-x_{n+2}=0$ at a finite number of isolated points $t_{1}, t_{2}, \ldots, t_{k}$. We have that the costate equations (B26) apply during $t \in\left[t_{0}, t_{1}\right)\left(t_{1}, t_{2}\right), \ldots,\left(t_{k}, t_{f}\right]$, and Eq. (A9) holds. Recognize that the inequality constraint (A6) in Appendix A is equivalent to that of inequality constraint (B12), so that $F(\boldsymbol{x})$ is simply $x_{n+1}$. Thus, $\partial F(\boldsymbol{x}) / \partial \boldsymbol{x}$ of Eq. (A10) is given by

$$
\frac{\partial F(\boldsymbol{x})}{\partial \boldsymbol{x}}=[0,0, \ldots, 0,1,0]^{T}
$$

and the jump conditions of Eq. (A10) are

$$
p_{n+1}^{+}=p_{n+1}^{-}+\mu_{i}
$$

where Eqs. (A12) and (A13) hold.

It is possible to simplify Eqs. (B26) and (B27) by recognizing Eq. (B29) implies

$$
\begin{gathered}
{\left[p_{n+1}(t+T)-p_{n+1}(t)\right]=\mu_{i}, \quad t_{i}-T<t \leq t_{i}} \\
{\left[p_{n+1}(t+T)-p_{n+1}(t)\right]=0, \quad \text { else }}
\end{gathered}
$$

This result is significant because it indicates that the necessary conditions switch between two optimization problems: For each isolated maximum occurring at $t_{i}$, while $t_{i}-T<t \leq t_{i}$, Eqs. (B26) and (B27) are

$$
\begin{gathered}
\dot{\boldsymbol{p}}=-\frac{\partial\left[\boldsymbol{p}^{T} \boldsymbol{f}(\boldsymbol{x}, u)\right]}{\partial \boldsymbol{x}}+\frac{\mu_{i}}{T} \frac{\partial \rho[\boldsymbol{x}(t), u(t)]}{\partial \boldsymbol{x}} \\
\frac{\partial\left[\boldsymbol{p}^{T} \boldsymbol{f}(\boldsymbol{x}, u)\right]}{\partial \boldsymbol{u}}=\frac{\mu_{i}}{T} \frac{\partial \rho[\boldsymbol{x}(t), u(t)]}{\partial \boldsymbol{u}}
\end{gathered}
$$

On the other hand, when outside of all intervals $\left(t_{i}-T, t_{i}\right]$, Eqs. (B26) and (B27) become

$$
\begin{gathered}
\dot{\boldsymbol{p}}=-\frac{\partial\left[\boldsymbol{p}^{T} \boldsymbol{f}(\boldsymbol{x}, u)\right]}{\partial \boldsymbol{x}} \\
\frac{\partial\left[\boldsymbol{p}^{T} \boldsymbol{f}(\boldsymbol{x}, u)\right]}{\partial \boldsymbol{u}}=0
\end{gathered}
$$

The interpretation of these two optimization problems is that, from $t_{i}-T$ to $t_{i}$, the solution is equivalent to the standard Lagrange optimal control problem,

$$
\min _{u} \frac{\mu_{i}}{T} \int_{t-T}^{t} \rho[x(\tau), u(\tau)] \mathrm{d} \tau
$$

which would result in an equivalent Hamiltonian,

$$
H_{1}=\boldsymbol{p}^{T} \boldsymbol{f}(\boldsymbol{x}, u)-\left(\mu_{i} / T\right) \rho[\boldsymbol{x}(t), u(t)]
$$

where the subscript 1 on $H$ indicates that it would apply to the first optimization problem. 
The second optimization problem occurs when not in the interval $\left(t_{i}-T, t_{i}\right]$. For this problem, the necessary conditions do not contain the cost function $\rho[x(t), u(t)]$ and would result in an equivalent Hamilonian,

$$
H_{2}=\boldsymbol{p}^{T} \boldsymbol{f}(\boldsymbol{x}, u)
$$

where the subscript 2 on $H$ indicates that it would apply to the second optimization problem. Note that this Hamiltonian is that of a minimum-time problem with interior-point constraints at times $t_{i}-T$ and $t_{i}$. Because Eqs. (B34) and (B35) do not contain the cost function $\rho[\boldsymbol{x}(t), u(t)]$, singular solutions are possible.

It is possible to consider the case that $F(\boldsymbol{x})$ attains it maximum over some finite intervals $\left(t_{1}, t_{1}^{\prime}\right), \ldots,\left(t_{k}, t_{k}^{\prime}\right)$, as in Appendix A for systems without time delay. Details may be found in Ref. 27, but will not be presented in this paper.

As has been shown, the necessary conditions for the minimax of a moving average functional are natural extensions of those that apply to a functional without time delay.

For the case of an isolated maximum, the necessary conditions can be summarized by the following observations.

1) For $t \in\left(t_{i}-T, t_{i}\right]$, where $t_{i}$ is the time that an isolated maximum occurs, the quantity $\left[p_{n+1}(t+T)-p_{n+1}(t)\right]=\mu_{i}$ and the necessary conditions are identical to those of a Lagrange optimal control problem with fixed boundary conditions.

2) For $t$ not in an interval $\left(t_{i}-T, t_{i}\right]$, the cost function is not considered in the equations for necessary conditions.

\section{Acknowledgments}

The authors would like to thank both the editor and reviewer for their guidance and advice.

\section{References}

${ }^{1}$ Hebert, J. M., “Air Vehicle Path Planning,” Ph.D. Dissertation, School of Engineering and Management, U.S. Air Force Inst. of Technology, WrightPatterson AFB, OH, Nov. 2001.

${ }^{2}$ Hebert, J., Jacques, D., Novy, M., and Pachter, M., "Cooperative Control of UAVS," AIAA Paper 2001-4240, Aug. 2001.

${ }^{3}$ Pachter, M., and Hebert, J., "Optimal Aircraft Trajectories for Radar Exposure Minimization," Proceedings of the American Control Conference, American Automatic Control Council, Dayton, OH, 2001, pp. 2365-2369.

${ }^{4}$ Judd, K. B., and McLain, T. W., "Spline Based Path Planning for Unmanned Air Vehicles," AIAA Paper 2001-4238, Aug. 2001.

${ }^{5}$ Chandler, P. R., Pachter, M., and Rasmussen, S., "UAV Cooperative Control," Proceedings of the American Control Conference, American Automatic Control Council, Dayton, OH, 2001.

${ }^{6}$ Chandler, P. R., Rasmussen, S., and Pachter, M., "UAV Cooperative Path Planning," AIAA Paper 2001-4238, Aug. 2000.

${ }^{7}$ Beard, R. W., McLain, T. W., Goodrich, M. A., and Anderson, E. P., "Coordinated Target Assignment and Intercept for Unmanned Air Vehicles," IEEE Journal of Robotics and Automation, Vol. 18, No. 6, 2002, pp. 911-922.

${ }^{8}$ Vian, J. L., and Moore, J. R., "Trajectory Optimization with Risk Minimization for Military Aircraft," Journal of Guidance, Control, and Dynamics, Vol. 12, No. 3, 1989, pp. 311-317.

${ }^{9}$ Rao, N. S., Fu, S. J., and Phillips, N. L., "Singular Perturbation Based Aircraft Trajectory Optimization for Threat Avoidance," Proceedings of the American Control Conference, American Automatic Control Council, Dayton, OH, 1990, pp. 314, 315.

${ }^{10}$ Rao, N. S., Phillips, N. L., Fu, S. J., and Conardy, N. M., "Horizontal Plane Trajectory Optimization for Threat Avoidance and Waypoint Rendezvous," Proceedings of the IEEE 1990 National Aerospace and Electronics Conference (NAECON), IEEE Publications, Piscataway, NJ, 1990, pp. 580-583.

${ }^{11}$ Godbole, D., Samad, T., and Gopal, V., "Active Multi-Model Control for Dynamic Maneuver Optimization of Unmanned Air Vehicles," Proceedings of the IEEE International Conference on Robotics and Automation, IEEE Publications, Piscataway, NJ, 2000, pp. 1257-1262.

${ }^{12}$ McFarland, M. B., Zachery, R. A., and Taylor, B. K., "Motion Planning for Reduced Observability of Autonomous Aerial Vehicles," Proceedings of the IEEE International Conference on Control Applications, IEEE Publications, Piscataway, NJ, 1999, pp. 231-235.
${ }^{13}$ Moore, F. W., "Radar Cross-Section Reduction Via Route Planning and Intelligent Control," IEEE Transactions on Control Systems Technology, Vol. 10, No. 5, 2002, pp. 696-700.

${ }^{14}$ Bortoff, S. A., "Path Planning for UAVs," Proceedings of the American Control Conference, American Automatic Control Council, Dayton, $\mathrm{OH}$, 2000, pp. 364-368.

${ }^{15}$ Misovec, K., Inanc, T., Wohletz, J., and Murray, R. M., "LowObservable Nonlinear Trajectory Generation for Unmanned Air Vehicles," Proceedings of the 42nd IEEE Conference on Decision and Control, IEEE Publications, Piscataway, NJ, 2003, pp. 3103-3110.

${ }^{16}$ Stone, W. R. (ed.), Radar Cross Sections of Complex Objects, IEEE Press, New York, pp. 7-29, 47-53.

${ }^{17}$ Yousef, N. N., "Radar Cross Section of Complex Targets," IEEE Proceedings, Vol. 77, No. 5, 1989, pp. 722-734.

${ }^{18}$ Mahafza, B. R., Radar Systems Analysis and Design Using MATLAB, Chapman and Hall, New York, 2002, pp. 85-101.

${ }^{19}$ Skolnik, M. I., Introduction to Radar Systems, McGraw-Hill, New York, 2001, pp. 33-49.

${ }^{20}$ Wilson, J. D., "Probability of Detecting Aircraft Targets," IEEE Transactions on Aerospace and Electronic Systems, Vol. 8, No. 6, 1972, pp. 757-761.

${ }^{21}$ North, D. O., "An Analysis of the Factors Which Determine Signal/ Noise Discrimination in Pulsed Carrier Systems," IEEE Proceedings, Vol. 51, No. 7, 1963, pp. 1015-1027.

${ }^{22}$ Bar-Shalom, Y., and Li, X. R., Estimation and Tracking, Principles, Techniques, and Software, Yaakov Bar-Shalom (YBS), New York, 1998, pp. 466-481.

${ }^{23} \mathrm{Li}, \mathrm{X} . \mathrm{R}$., and Bar-Shalom, Y., "Detection Threshold Selection for Tracking Performance Optimization,' IEEE Transactions on Aerospace and Electronic Systems, Vol. 30, No. 3, 1994, pp. 742-749.

${ }^{24} \mathrm{Li}, \mathrm{X} . \mathrm{R}$., and Bar-Shalom, Y., "Performance Prediction of the Interacting Multiple Model Algorithm," IEEE Transactions an Aerospace and Electronic Systems, Vol. 29, No. 3, 1993, pp. 755-771.

${ }^{25}$ Ben-Asher, J. Z., and Yaesh, I., Advances in Missile Guidance Theory, Vol. 180, Progress in Astronautics and Aeronautics, AIAA, Reston, VA, 1998, pp. 1-5.

${ }^{26}$ Shaw, R. L., Fighter Combat, Tactics and Maneuvering, Naval Inst. Press, U.S. Naval Inst., Annapolis, MD, 1985, p. 5.

${ }^{27}$ Kabamba, P., Meerkov, S., and Zeitz, F., "UCAV Path Planning in the Presence of Radar-Guided Surface-to-Air Missile Threats,' Technical Rept. CGR 04-14, Electrical Engineering and Computer Science Systems, Univ. of Michigan, Ann Arbor, MI, Oct. 2004.

${ }^{28}$ Nezavisimoe Voennoe Obozrenie, No. 12 (135), 2-8 April 1999, p. 3 .

${ }^{29}$ Krasnaya Zvezda, 31 March 1999, p. 1.

${ }^{30}$ Bryson, A. E., and Ho, Y.-C., Applied Optimal Control, Optimization, Estimation, and Control, Hemisphere, Washington, DC, 1981, pp. 110-117, 247-250.

${ }^{31}$ Lu, P., "Minimax and Maximax Optimal Control Problems with Applications in Aerospace Engineering," Ph.D. Dissertation, Dept. of Aerospace Engineering, Univ. of Michigan, Ann Arbor, MI, 1988.

${ }^{32}$ Lu, P., and Vinh, N. X., "Optimal Control Problems with Maximum Functional," Journal of Guidance, Control, and Dynamics, Vol. 14, No. 6, 1991, pp. 1215-1223.

${ }^{33}$ Lu, P., and Vinh, N. X., "On the Minimax Optimal Control Problem and Its Variations," Optimal Control: Calculus of Variations, Optimal Control Theory, and Numerical Methods, edited by R. Burlisch, A. Miele, J. Stoer, and K. H. Well, Vol. III, International Series of Numerical Mathematics, Birkhäuser Verlag, Berlin, 1993, pp. 99-112.

${ }^{34}$ Vinh, N. X., and Lu, P., "Necessary Conditions for Maximax Problems with Application to Aeroglide of Hypervelocity Vehicles," Acta Astronautica, Vol. 15, No. 6, 1987, pp. 413-420.

${ }^{35}$ Lu, P., and Vinh, N. X., "Minimax Optimal Control for Atmospheric FlyThrough Trajectories," Journal of Optimization Theory and Applications, Vol. 57, No. 1, 1988, pp. 41-58.

${ }^{36}$ Lee, E. B., and Markus, L., Foundation of Optimal Control Theory, Wiley, New York, 1966, pp. 259-265.

${ }^{37}$ Pontryagin, L. S., Boltyanskii, V. G., Gramkreledze, Q. V., and Mishchenko, E. F., The Mathematical Theory of Optimal Processes, Interscience, New York, 1962, pp. 257-316.

${ }^{38}$ Bunce, G. R., "A Maximum Principle for Time-Lag Control Problems with Bounded State," Journal of Optimization Theory and Applications, Vol. 22, No. 4, 1977, pp. 563-606.

${ }^{39}$ Malek-Zavarei, M., and Jamshidi, M., Time-Delay Systems: Analysis, Optimization and Applications, North-Holland, Amsterdam, 1987, pp. $155-160$. 